\title{
Introduction to Minitrack on Society, Information, Technology and Economics
}

\author{
Eric K. Clemons, Rajiv Dewan, Robert J. Kauffman and Thomas A. Weber
}

Emerging technologies and the ubiquitous processing of all kinds of information - personal and private, governmental and public - have created new problems and dilemmas in society, thus requiring fresh approaches in both theory and observation.

The 2016 SITE Mini-Track begins with a session on "IT, Trust and Strategy." The first paper, by Sunghun Chung, Kunsoo Han, Animesh Animesh, and Alain Pinsonneault, is "Competitive Impacts of Software Patents in the IT Industry." It explores the business value of IT innovation to study how a firm's software patents' affect the market value of its rivals in the IT industries in the U.S. Next is Eric K. Clemons' paper on "Online Trust: An International Study of Subjects' Willingness to Shop at Online Merchants, Including the Effects of Promises and of Third-Party Guarantees." This is a continuing theme in his SITE MiniTrack contributions, involving comparisons of consumers in the U.S., China, Germany and Singapore, based on simultaneous lab experiments. The treatments involve promises of quality and authenticity (or none at all), some backed by third-party assurance providers, with sellers of different risk levels. He reports that Chinese consumers trust their best online vendors very well, while U.S. consumers now are viewing online shopping and physical shopping with similar degrees of trust. The third paper, on IT strategy, is "Versioning of Video Games: Go Vertical in a Horizontal Market? by Deb Dey and Atanu Lahiri. The authors discuss the design of downloadable content for experience goods, and emphasize why manufacturers may want to vertically differentiate themselves: the base version may serve as a teaser that helps consumers learn about the full-scale version of a video game.

The second session is entitled "Cloud Pricing, Innovation Tournaments and the Sharing Economy." It showcases a range of contemporary issues that have been discussed over the years in this mini-track. The first paper is by Hemant K. Bhargava and Manish Gangwar, who ask: "Go with 'Pay As You Go' or 'All You Can Eat'? Simple Pricing for Computing and Information Services." The authors assess the efficacy of unit pricing, buffet pricing, and hybrid three-part tariffs for the offering of SaaS and IT services in terms of profitability and efficiency. A study on Kaggle, team capabilities, and "Winning Strategies in Dynamic
Innovation Tournaments" follows. Indika Dissanayake, Jie Zhang and Mahmut Yasar estimate the effects of team strategies and mergers on their probability of winning through the provision of creative solutions to the problems that are posed. The session ends with Thomas A. Weber's modeling work on "Optimal Retail in a Sharing Economy." The author characterizes a retailer's optimal pricing strategy with and without sharing markets, and finds that retailers have an incentive to encourage sharing for high-cost products, whereas low-cost products are most profitably sold without sharing. His dynamic model suggests that sharing markets are likely to lead to higher prices for new products and that prices for sharing track retail prices. The presence of sharing tends to change the price elasticity of demand, and this may work for or against the retailers.

The third session is on "Connectivity, Social Networks and Third-Party Payer Business Models." It opens with a paper by Matthias Berger, Christian Matt, and Thomas Hess that explores the question: "Connectivity Is Ubiquitous, But Is It Beneficial? A Numerical Approach to Assess Individuals' Valuations of Digital Physical Systems." The authors' study valuation and selection of components in home technology networks and the network effects of connectivity. They use combinatorial optimization to optimize user network configuration. The next paper is our best-paper nominee, "Strategic Network Formation in a Location-Based Social Network: A Topic Modeling Approach," by Gene Moo Lee, Liangfei Qiu, and Andrew B. Whinston. They analyze a structural model of link creation with user network proximity, geography, mobility, and SMSs. They also provide topic models with Gowalla check-in data to assess how homophily affects network development. Finally, Eric K. Clemons and Joshua Wilson present "Modeling Competition in Mandatory Participation Third-Party Payer Business Models: The Complex Case of Sponsored Search." They report on how platform operators are able to leverage parallel monopolies, and free themselves from the pressures of the market, so that competition does not result in price decreases or quality increases.

The mini-track will conclude with an open discussion and debate about the highlights, ideas, and solutions that were presented and discussed over the course 
of the mini-track sessions. Each year, we engage in this process to encourage next steps and further study of important business, social and policy issues, as well as to collaboratively identify new directions for research. 